\title{
Complexity of Banach space valued and parametric integration
}

\author{
Thomas Daun, Stefan Heinrich \\ Department of Computer Science \\ University of Kaiserslautern \\ D-67653 Kaiserslautern, Germany \\ e-mail: [daun,heinrich]@informatik.uni-kl.de
}

\begin{abstract}
We study the complexity of Banach space valued integration. The input data are assumed to be $r$-smooth. We consider both definite and indefinite integration and analyse the deterministic and the randomized setting. We develop algorithms, estimate their error, and prove lower bounds. In the randomized setting the optimal convergence rate turns out to be related to the geometry of the underlying Banach space.

Then we study the corresponding problems for parameter dependent scalar integration. For this purpose we use the Banach space results and develop a multilevel scheme which connects Banach space and parametric case.
\end{abstract}

\section{Introduction}

While complexity of integration in the scalar case is well-studied, the Banach space case has not been investigated before. We consider both definite and indefinite integration, develop randomized algorithms and analyse their convergence. We also prove lower bounds and this way estimate the complexity of the integration problems. The results are related to the geometry of the underlying Banach space. It turns out that the bounds are matching and the algorithms are of optimal order for special spaces, including the $L_{p}$ spaces. For general Banach spaces an arbitrarily small gap in the exponent of upper and lower bounds remains. We also study the deterministic case and show that for arbitrary Banach spaces our methods are of optimal order for any fixed choice of the random parameters.

The study of Banach space valued problems turns out to be crucial for the development of algorithms and the complexity analysis for parameter dependent problems, since such problems can be viewed as special cases of this general context. To apply our Banach space results we need a way of passing from 
Banach space valued to scalar information (function values). This is achieved by a multilevel scheme which is based on the ideas of $[2,6]$. As a result, we obtain multilevel algorithms for the parametric problems and show that they are of optimal order (in some cases up to a logarithmic factor).

The paper is organized as follows. In Section 2 we provide the needed notation and technical tools. Section 3 contains algorithms for definite and indefinite Banach space valued integration, their analysis and lower bounds. In Section 4 we present the multilevel approach and in Section 5 we apply the previous results to the parametric problems.

\section{Preliminaries}

Let $\mathbb{N}=\{1,2, \ldots\}$ and $\mathbb{N}_{0}=\{0,1,2, \ldots\}$. We introduce some notation and concepts from Banach space theory needed in the sequel. For a Banach space $X$ the closed unit ball is denoted by $B_{X}$, the identity mapping on $X$ by $I_{X}$, and the dual space by $X^{*}$. Given another Banach space $Y$, we let $\mathscr{L}(X, Y)$ be the space of bounded linear mappings $T: X \rightarrow Y$ endowed with the canonical norm. If $X=Y$, we write $\mathscr{L}(X)$ instead of $\mathscr{L}(X, X)$. Throughout the paper the norm of $X$ is denoted by $\|\cdot\|$. Other norms are usually distinguished by subscripts. We assume all considered Banach spaces to be defined over the same scalar field $\mathbb{K}=\mathbb{R}$ or $\mathbb{K}=\mathbb{C}$.

Let $Q=[0,1]^{d}$ and let $C^{r}(Q, X)$ be the space of all $r$-times continuously differentiable functions $f: Q \rightarrow X$ equipped with the norm

$$
\|f\|_{C^{r}(Q, X)}=\max _{0 \leq j \leq r, t \in Q}\left\|f^{(j)}(t)\right\| .
$$

For $r=0$ we write $C^{0}(Q, X)=C(Q, X)$, which is the space of continuous $X$ valued functions on $Q$. If $X=\mathbb{K}$, we write $C^{r}(Q)$ and $C(Q)$.

Let $1 \leq p \leq 2$. A Banach space $X$ is said to be of (Rademacher) type $p$, if there is a constant $c>0$ such that for all $n \in \mathbb{N}$ and $x_{1}, \ldots, x_{n} \in X$

$$
\mathbb{E}\left\|\sum_{i=1}^{n} \varepsilon_{i} x_{i}\right\|^{p} \leq c^{p} \sum_{k=1}^{n}\left\|x_{i}\right\|^{p},
$$

where $\left(\varepsilon_{i}\right)_{i=1}^{n}$ is a sequence of independent Bernoulli random variables with $\mathbb{P}\left\{\varepsilon_{i}=\right.$ $-1\}=\mathbb{P}\left\{\varepsilon_{i}=+1\right\}=1 / 2$ (we refer to $[9,7]$ for this notion and related facts). The smallest constant satisfying (1) is called the type $p$ constant of $X$ and is denoted by $\tau_{p}(X)$. If there is no such $c>0$, we put $\tau_{p}(X)=\infty$. The space $L_{p_{1}}(\mathcal{N}, \nu)$ with $(\mathcal{N}, \nu)$ an arbitrary measure space and $p_{1}<\infty$ is of type $p$ with $p=\min \left(p_{1}, 2\right)$. Furthermore, there is a constant $c>0$ such that $\tau_{2}\left(\ell_{\infty}^{n}\right) \leq c(\log (n+1))^{1 / 2}$ for all $n \in \mathbb{N}$. We will use the following result (see [7], Prop. 9.11). 
Lemma 1. Let $1 \leq p \leq 2$, let $X$ be a Banach space, $n \in \mathbb{N}$ and $\left(\theta_{i}\right)_{i=1}^{n}$ be a sequence of independent $X$-valued random variables with $E\left\|\theta_{i}\right\|^{p}<\infty$ and $\mathbb{E} \theta_{i}=0(i=1, \ldots, n)$. Then

$$
\left(\mathbb{E}\left\|\sum_{i=1}^{n} \theta_{i}\right\|^{p}\right)^{1 / p} \leq 2 \tau_{p}(X)\left(\sum_{k=1}^{n} \mathbb{E}\left\|\theta_{i}\right\|^{p}\right)^{1 / p} .
$$

We need some notation and facts on tensor products of Banach spaces. For details and proofs we refer to [1] and [8]. Let $X \otimes Y$ be the algebraic tensor product of Banach spaces $X$ and $Y$. For $z=\sum_{i=1}^{n} x_{i} \otimes y_{i} \in X \otimes Y$ define

$$
\lambda(z)=\sup _{u \in B_{X^{*}}, v \in B_{Y^{*}}}\left|\sum_{i=1}^{n}\left\langle x_{i}, u\right\rangle\left\langle y_{i}, v\right\rangle\right| .
$$

The injective tensor product $X \otimes_{\lambda} Y$ is defined as the completion of $X \otimes Y$ with respect to the norm $\lambda$. We use the canonical isometric identification

$$
C(Q, X)=X \otimes_{\lambda} C(Q)
$$

valid for arbitrary Banach spaces $X$, and in particular, for $d>1$

$$
C\left([0,1]^{d}\right)=C([0,1]) \otimes_{\lambda} C\left([0,1]^{d-1}\right)=C([0,1]) \otimes_{\lambda} \cdots \otimes_{\lambda} C([0,1]) .
$$

Given Banach spaces $X_{1}, X_{2}, Y_{1}, Y_{2}$ and operators $T_{1} \in \mathscr{L}\left(X_{1}, Y_{1}\right), T_{2} \in \mathscr{L}\left(X_{2}, Y_{2}\right)$, the algebraic tensor product $T_{1} \otimes T_{2}: X_{1} \otimes X_{2} \rightarrow Y_{1} \otimes Y_{2}$ extends to a bounded linear operator $T_{1} \otimes T_{2} \in \mathscr{L}\left(X_{1} \otimes_{\lambda} X_{2}, Y_{1} \otimes_{\lambda} Y_{2}\right)$ with

$$
\left\|T_{1} \otimes T_{2}\right\|_{\mathscr{L}\left(X_{1} \otimes_{\lambda} X_{2}, Y_{1} \otimes_{\lambda} Y_{2}\right)}=\left\|T_{1}\right\|_{\mathscr{L}\left(X_{1}, Y_{1}\right)}\left\|T_{2}\right\|_{\mathscr{L}\left(X_{2}, Y_{2}\right)} .
$$

For $r, m \in \mathbb{N}$ we let $P_{m}^{r, 1} \in \mathscr{L}(C([0,1]))$ be composite with respect to the partition of $[0,1]$ into $m$ intervals of length $m^{-1}$ Lagrange interpolation of degree $r$. Let

$$
P_{m}^{r, d}=\otimes^{d} P_{m}^{r, 1} \in \mathscr{L}\left(C\left([0,1]^{d}\right)\right)
$$

be its $d$-dimensional version. Setting $\Gamma_{k}^{d}=\left\{\frac{i}{k}: 0 \leq i \leq k\right\}^{d}$ for $k \in \mathbb{N}$, it follows that $P_{m}^{r, d}$ interpolates on $\Gamma_{r m}^{d}$. Given a Banach space $X$, the $X$-valued versions of the operators above are defined in the sense of identification (2) as

$$
P_{m}^{r, d, X}=I_{X} \otimes P_{m}^{r, d} .
$$

This means that if $P_{m}^{r, d}$ is represented as

$$
P_{m}^{r, d} f=\sum_{s \in \Gamma_{r m}^{d}} f(s) \varphi_{s} \quad(f \in C(Q))
$$


for some $\varphi_{s} \in C(Q)$, then $P_{m}^{r, d, X}$ has the representation

$$
P_{m}^{r, d, X} f=\sum_{s \in \Gamma_{r m}^{d}} f(s) \varphi_{s} \quad(f \in C(Q, X)) .
$$

We can obviously consider $P_{m}^{r, d, X}$ also as an operator from $\ell_{\infty}\left(\Gamma_{r m}^{d}, X\right)$ to $C(Q, X)$. Given $r \in \mathbb{N}_{0}$ and $d \in \mathbb{N}$, there are constants $c_{1}, c_{2}>0$ such that for all Banach spaces $X$ and all $m \in \mathbb{N}$

$$
\left\|P_{m}^{r, d, X}\right\|_{\mathscr{L}(C(Q, X))} \leq c_{1}, \quad \sup _{f \in B_{C^{r}(Q, X)}}\left\|f-P_{m}^{r, d, X} f\right\|_{C(Q, X)} \leq c_{2} m^{-r} .
$$

The scalar case of (5) is well-known, which in turn readily implies the Banach space case by considering functions $f_{u} \in C(Q)$ given for $f \in C(Q, X)$ and $u \in$ $B_{X^{*}}$ by $f_{u}(t)=\langle f(t), u\rangle(t \in Q)$.

We will work in the setting of information-based complexity theory (IBC), see $[12,10]$. For the precise notions used here we also refer to [3, 4]. An abstract numerical problem is described by a tuple $\mathscr{P}=(F, G, S, K, \Lambda)$. The set $F$ is the set of input data, $G$ is a normed linear space and $S: F \rightarrow G$ an arbitrary mapping, the solution operator, which maps the input $f \in F$ to the exact solution $S f . K$ is an arbitrary set and $\Lambda$ is a set of mappings from $F$ to $K$ - the class of admissible information functionals.

A randomized algorithm for $\mathscr{P}$ is a family $A=\left(A_{\omega}\right)_{\omega \in \Omega}$, where $(\Omega, \Sigma, \mathbb{P})$ is the underlying probability space and each $A_{\omega}$ is a mapping $A_{\omega}: F \rightarrow G$. For $\omega$ fixed, $A_{\omega}: F \rightarrow G$ is a deterministic algorithm, that is, stands for a deterministic process (depending on $\omega$ ) which uses values of information functionals on $f \in F$ in an adaptive way. The result of the algorithm, $A_{\omega} f$, is the approximation to $S f$. The parameter $\omega$ incorporates all randomness used in the algorithm $A=\left(A_{\omega}\right)_{\omega \in \Omega}$. The error of $A$ is defined as

$$
e(S, A, F)=\sup _{f \in F} \mathbb{E}\left\|S f-A_{\omega} f\right\|_{G} .
$$

Let $\operatorname{card}\left(A_{\omega}, f\right)$ be the number of information functionals used by $A_{\omega}$ at input $f$. We define the cardinality of $A$ as

$$
\operatorname{card}(A, F)=\sup _{f \in F} \mathbb{E} \operatorname{card}\left(A_{\omega}, f\right) .
$$

The central notion of IBC is the $n$-th minimal error, which is defined for $n \in \mathbb{N}_{0}$ as

$$
e_{n}^{\mathrm{ran}}(S, F)=\inf _{\operatorname{card}(A, F) \leq n} e(S, A, F) .
$$

So $e_{n}^{\text {ran }}(S, F)$ is the minimal possible error among all randomized algorithms that use (on the average) at most $n$ information functionals.

We can introduce respective notions for the deterministic setting as a special case of the above by considering only one-point probability spaces $\Omega=\left\{\omega_{0}\right\}$, 
which means that there is no dependence on randomness. Let $e_{n}^{\operatorname{det}}(S, F)$ denote the $n$-th minimal error in this setting.

The complexity of definite scalar integration has been studied in numerous papers, see $[12,10,11]$ and the references therein. The complexity of scalar indefinite integration was considered only recently in [5]. Let us summarize these known results.

Let $r \in \mathbb{N}_{0}, \iota \in\{0,1\}$, and let $S_{\iota}$ be the operator of definite $(\iota=0)$, respectively indefinite $(\iota=1)$ scalar integration (for the precise definitions see $(8-9)$ and the line after (10)). Then there are constants $c_{1-4}>0$ such that for $n \in \mathbb{N}$ the following hold. The deterministic $n$-th minimal error satisfies

$$
c_{1} n^{-r / d} \leq e_{n}^{\operatorname{det}}\left(S_{\iota}, B_{C^{r}(Q)}\right) \leq c_{2} n^{-r / d}
$$

while the randomized $n$-th minimal errors fulfills

$$
c_{3} n^{-r / d-1 / 2} \leq e_{n}^{\operatorname{ran}}\left(S_{\iota}, B_{C^{r}(Q)}\right) \leq c_{4} n^{-r / d-1 / 2} .
$$

The Banach space cases of both problems have not been studied before. The complexity of parametric definite integration was analysed in [6] (this result is stated as part of Theorem 2 below), parametric indefinite integration has not been investigated before.

Throughout the paper $c, c_{1}, c_{2}, \ldots$ are constants, which depend only on the problem parameters $r, d$, but depend neither on the algorithm parameters $n, l$ etc. nor on the input $f$. We emphasize that they do not depend on $X$ either. The same symbol may denote different constants, even in a sequence of relations.

\section{Banach space valued integration}

Let $X$ be a Banach space, $r \in \mathbb{N}_{0}$, and let the definite integration operator $S_{0}^{X}: C(Q, X) \rightarrow X$ be given by

$$
S_{0}^{X} f=\int_{Q} f(t) d t
$$

Put $F=B_{C^{r}(Q, X)}, G=X$, let $K=X$ and $\Lambda=\Lambda(Q, X)=\left\{\delta_{t}: t \in Q\right\}$ with $\delta_{t}(f)=f(t)$. So here we consider $X$-valued information functionals. This describes the definite integration problem

$$
\mathscr{P}_{0}=\left(B_{C^{r}(Q, X)}, X, S_{0}^{X}, X, \Lambda(Q, X)\right) .
$$

The indefinite integration operator $S_{1}^{X}: C(Q, X) \rightarrow C(Q, X)$ is given by

$$
\left(S_{1}^{X} f\right)(t)=\int_{[0, t]} f(u) d u \quad(t \in Q),
$$


with $[0, t]=\prod_{i=1}^{d}\left[0, t_{i}\right]$ for $t=\left(t_{i}\right)_{i=1}^{d} \in Q$. Here we take $G=C(Q, X)$, while $F, K$, and $\Lambda$ are the same as above, so the indefinite integration problem is

$$
\mathscr{P}_{1}=\left(B_{C^{r}(Q, X)}, C(Q, X), S_{1}^{X}, X, \Lambda(Q, X)\right) .
$$

Note that in the sense of identification (2) we have

$$
S_{\iota}^{X}=I_{X} \otimes S_{\iota} \quad(\iota=0,1)
$$

where $S_{\iota}$ is the scalar version of $S_{\iota}^{X}$, with $X=\mathbb{K}$.

Now we present algorithms for the two integration problems (8) and (9). We start with definite integration. Let $n \in \mathbb{N}$ and let $\xi_{i}: \Omega \rightarrow Q(i=1, \ldots, n)$ be independent, uniformly distributed on $Q$ random variables on some complete probability space $(\Omega, \Sigma, \mathbb{P})$. Set for $f \in C(Q, X)$

$$
A_{n, \omega}^{0,0, X} f=\frac{1}{n} \sum_{i=1}^{n} f\left(\xi_{i}(\omega)\right)
$$

and, if $r \geq 1$, put $k=\left\lceil n^{1 / d}\right\rceil$ and

$$
A_{n, \omega}^{0, r, X} f=S_{0}^{X}\left(P_{k}^{r, d, X} f\right)+A_{n, \omega}^{0,0, X}\left(f-P_{k}^{r, d, X} f\right) .
$$

We write $A_{n, \omega}^{0, r}$ for the scalar case $A_{n, \omega}^{0, r, \mathbb{K}}$. Finally we set $A_{n}^{0, r, X}=\left(A_{n, \omega}^{0, r, X}\right)_{\omega \in \Omega}$. In the scalar case for $r=0$ this is just the standard Monte Carlo method and for $r \geq 1$ the Monte Carlo method with separation of the main part. Note that for $r \in \mathbb{N}_{0}, n \in \mathbb{N}, \omega \in \Omega$

$$
A_{n, \omega}^{0, r, X}=I_{X} \otimes A_{n, \omega}^{0, r} .
$$

Let us turn to the error analysis for this algorithm. Fixing the random parameter $\omega \in \Omega$ means that we obtain a deterministic method, the error of which we also consider.

Proposition 1. Let $r \in \mathbb{N}_{0}$ and $1 \leq p \leq 2$. Then there are constants $c_{1-3}>0$ such that for all Banach spaces $X, n \in \mathbb{N}, \omega \in \Omega$ we have $\operatorname{card}\left(A_{n, \omega}^{0, r, X}\right) \leq c_{1} n$ and for all $f \in C^{r}(Q, X)$

$$
\begin{aligned}
\left\|S_{0}^{X} f-A_{n, \omega}^{0, r, X} f\right\| & \leq c_{2} n^{-r / d}\|f\|_{C^{r}(Q, X)} \\
\left(\mathbb{E}\left\|S_{0}^{X} f-A_{n, \omega}^{0, r, X} f\right\|^{p}\right)^{1 / p} & \leq c_{3} \tau_{p}(X) n^{-r / d-1+1 / p}\|f\|_{C^{r}(Q, X)} .
\end{aligned}
$$

Proof. Let $r=0$ and $f \in C(Q, X)$. With

$$
\eta_{i}(\omega)=\int_{Q} f(t) d t-f\left(\xi_{i}(\omega)\right)
$$

we have $\mathbb{E} \eta_{i}(\omega)=0$,

$$
S_{0}^{X} f-A_{n, \omega}^{0,0, X} f=\frac{1}{n} \sum_{i=1}^{n} \eta_{i}(\omega)
$$


and

$$
\left\|\eta_{i}(\omega)\right\| \leq 2\|f\|_{C(Q, X)} .
$$

This implies (14) and, together with Lemma 1, also (15). The case $r \geq 1$ follows directly from the case $r=0$ and relation (5), since

$$
S_{0}^{X} f-A_{n, \omega}^{0, r, X} f=S_{0}^{X}\left(f-P_{k}^{r, d, X} f\right)-A_{n, \omega}^{0,0, X}\left(f-P_{k}^{r, d, X} f\right) .
$$

Next we consider indefinite integration. First we assume $r=0$ and present the Banach space version of the algorithm from Section 4 of [5]. It is a combination of the Smolyak algorithm with the Monte Carlo method. Fix any $m \in \mathbb{N}, m \geq 2$ and $L \in \mathbb{N}_{0}$. For $\bar{l}=\left(l_{1}, \ldots, l_{d}\right) \in \mathbb{N}_{0}^{d}$ we set $|\bar{l}|=l_{1}+\cdots+l_{d}$ and define $U_{\bar{l}}, V_{L} \in \mathscr{L}(C(Q))$ by

$$
U_{\bar{l}}=\left(P_{m^{l_{1}}}^{1,1}-P_{m^{l_{1}-1}}^{1,1}\right) \otimes \cdots \otimes\left(P_{m^{l} d-1}^{1,1}-P_{m^{l} d-1}^{1,1}\right) \otimes P_{m^{l} d}^{1,1},
$$

with the understanding that $P_{m^{-1}}^{1,1}:=0$. Furthermore, put

$$
V_{L}=\sum_{\bar{l} \in \mathbb{N}_{0}^{d},|\bar{l}|=L} U_{\bar{l}}
$$

and let

$$
U_{\bar{l}}^{X}=I_{X} \otimes U_{\bar{l}}, \quad V_{L}^{X}=I_{X} \otimes V_{L}
$$

be the respective Banach space versions. Set

$$
\overline{1}=(\underbrace{1, \ldots, 1}_{d}), \quad m^{\bar{l}}=\left(m^{l_{1}}, \ldots, m^{l_{d}}\right), \quad \Gamma_{m^{\bar{l}}}=\Gamma_{m^{l_{1}}}^{1} \times \cdots \times \Gamma_{m^{l_{d}}}^{1},
$$

and for $\bar{i}=\left(i_{1}, \ldots, i_{d}\right) \in \mathbb{N}^{d}$ with $\overline{1} \leq \bar{i} \leq m^{\bar{l}}$ (component-wise inequalities)

$$
Q_{\bar{l}, \bar{i}}=\left[\frac{i_{1}-1}{m^{l_{1}}}, \frac{i_{1}}{m^{l_{1}}}\right] \times \cdots \times\left[\frac{i_{d}-1}{m^{l_{d}}}, \frac{i_{d}}{m^{l_{d}}}\right] .
$$

So $\left(Q_{\bar{l}, \bar{i}}\right)_{\overline{1}<\bar{i}<m^{\bar{l}}}$ is the partition of $Q$ corresponding to the grid $\Gamma_{m^{\bar{l}}}$. Let $\xi_{\bar{l}, \bar{i}}$ : $\Omega \rightarrow Q_{\bar{l}, \bar{i}}\left(|\bar{l}|=L, \overline{1} \leq \bar{i} \leq m^{\bar{l}}\right)$ be independent random variables on a complete probability space $(\Omega, \Sigma, \mathbb{P})$ such that $\xi_{\bar{l}, \bar{i}}$ is uniformly distributed on $Q_{\bar{l}, \bar{i}}$. Define $g_{\bar{l}, \omega} \in \ell_{\infty}\left(\Gamma_{m^{\bar{l}}}, X\right)$ by

$$
g_{\bar{l}, \omega}(t)=\sum_{\bar{j}: Q_{\bar{l}, \bar{j}} \subseteq[0, t]}\left|Q_{\bar{l}, \bar{j}}\right| f\left(\xi_{\bar{l}, \bar{j}}(\omega)\right) \quad\left(t \in \Gamma_{m^{\bar{l}}}\right),
$$

with the convention that $g_{\bar{l}, \omega}(t)=0$ if there is no $\bar{j}$ with $Q_{\bar{l}, \bar{j}} \subseteq[0, t]$ (that is, if some component of $t$ is zero). Finally we put

$$
L=2 d-1
$$


and, given $n \in \mathbb{N}$,

$$
m=\left\lceil(n+1)^{\frac{1}{L}}\right\rceil
$$

If $r=0$, we define

$$
A_{n, \omega}^{1,0, X} f:=\sum_{\bar{l} \in \mathbb{N}_{0}^{d},|\bar{l}|=L} U_{\bar{l}}^{X} g_{\bar{l}, \omega} .
$$

In the case $r \geq 1$ we put $k=\left\lceil n^{1 / d}\right\rceil$ and

$$
A_{n, \omega}^{1, r, X} f=S_{1}^{X}\left(P_{k}^{r, d, X} f\right)+A_{n, \omega}^{1,0, X}\left(f-P_{k}^{r, d, X} f\right) .
$$

Finally set $A_{n}^{1, r, X}=\left(A_{n, \omega}^{1, r, X}\right)_{\omega \in \Omega}$. Similarly to (13) we have for $r \in \mathbb{N}_{0}, n \in \mathbb{N}$, $\omega \in \Omega$

$$
A_{n, \omega}^{1, r, X}=I_{X} \otimes A_{n, \omega}^{1, r},
$$

with $A_{n, \omega}^{1, r}=A_{n, \omega}^{1, r, \mathbb{K}}$. The scalar case of the following result for $r=0$ has been shown in [5]. We use the tensor product technique to carry over parts of the proof.

Proposition 2. Let $r \in \mathbb{N}_{0}, 1 \leq p \leq 2$. Then there are constants $c_{1-3}>0$ such that for all Banach spaces $X, n \in \mathbb{N}, \omega \in \Omega$ we have $\operatorname{card}\left(A_{n, \omega}^{1, r, X}\right) \leq c_{1} n$ and for all $f \in C^{r}(Q, X)$

$$
\begin{aligned}
\left\|S_{1}^{X} f-A_{n, \omega}^{1, r, X} f\right\|_{C(Q, X)} & \leq c_{2} n^{-r / d}\|f\|_{C^{r}(Q, X)} \\
\left(\mathbb{E}\left\|S_{1}^{X} f-A_{n, \omega}^{1, r, X} f\right\|_{C(Q, X)}^{p}\right)^{1 / p} & \leq c_{3} \tau_{p}(X) n^{-r / d-1+1 / p}\|f\|_{C^{r}(Q, X)} .
\end{aligned}
$$

Proof. We start with the case $r=0$, where we have

$$
\begin{aligned}
& \left\|S_{1}^{X} f-A_{n, \omega}^{1,0, X} f\right\|_{C(Q, X)} \\
& \quad \leq\left\|S_{1}^{X} f-V_{L}^{X} S_{1}^{X} f\right\|_{C(Q, X)}+\left\|V_{L}^{X} S_{1}^{X} f-A_{n, \omega}^{1,0, X} f\right\|_{C(Q, X)} .
\end{aligned}
$$

The first term can be estimated using

$$
\left\|S_{1}^{X}-V_{L}^{X} S_{1}^{X}\right\|_{\mathscr{L}(C(Q, X))} \leq c m^{-L+d-1},
$$

the scalar case of which is Lemma 4.2 of [5]. The Banach space case follows by taking tensor products and using (10) and (18). Now we consider the second term. We have

$$
\left\|V_{L}^{X} S_{1}^{X} f-A_{n, \omega}^{1,0, X} f\right\|_{C(Q, X)} \leq \sum_{\bar{l} \in \mathbb{N}_{0}^{d},|\bar{l}|=L}\left\|U_{\bar{l}}^{X} S_{1}^{X} f-U_{\bar{l}}^{X} g_{\bar{l}, \omega}\right\|_{C(Q, X)}
$$

and

$$
\begin{aligned}
& \left\|U_{\bar{l}}^{X} S_{1}^{X} f-U_{\bar{l}}^{X} g_{\bar{l}, \omega}\right\|_{C(Q, X))} \\
& \quad \leq\left\|U_{\bar{l}}^{X}\right\|_{\mathscr{L}\left(\ell_{\infty}\left(\Gamma_{m^{\bar{l}}}\right), C(Q, X)\right)}\left\|\left.\left(S_{1}^{X} f\right)\right|_{\Gamma_{m^{\bar{l}}}}-g_{\bar{l}, \omega}\right\|_{\ell_{\infty}\left(\Gamma_{m^{\bar{l}}}\right)} \\
& \quad \leq c \max _{t \in \Gamma_{m^{\bar{l}}}}\left\|\int_{[0, t]} f(t) d t-\sum_{\bar{j}: Q_{\bar{l}, \bar{j}} \subseteq[0, t]}\left|Q_{\bar{l}, \bar{j}}\right| f\left(\xi_{\bar{l}, \bar{j}}\right)\right\|=c \max _{\overline{1} \leq \bar{i} \leq m^{\bar{l}}}\left\|\sum_{\overline{1} \leq \bar{j} \leq \bar{i}} \eta_{\bar{l}, \bar{j}}\right\|
\end{aligned}
$$


with

$$
\eta_{\bar{l}, \bar{j}}=\int_{Q_{\bar{l}, \bar{j}}} f(t) d t-\left|Q_{\bar{l}, \bar{j}}\right| f\left(\xi_{\bar{l}, \bar{j}}\right) \quad\left(\overline{1} \leq \bar{j} \leq m^{\bar{l}}\right) .
$$

The random variables $\left\{\eta_{\bar{l}, \bar{j}}: \overline{1} \leq \bar{j} \leq m^{\bar{l}}\right\}$ are independent, of mean zero, and satisfy

$$
\left\|\eta_{\bar{l}, \bar{j}}\right\| \leq 2\left|Q_{\bar{l}, \bar{j}}\right|\|f\|_{C(Q, X)}=2 m^{-L}\|f\|_{C(Q, X)} .
$$

Combining (20-21) and (27-32), we obtain (25) for $r=0$.

For $p>1$ we get from Lemma 4.3 of [5] (a simple generalization of Doob's inequality, the proof of which literally carries over to the Banach space case)

$$
\left(\mathbb{E} \max _{\overline{1} \leq \bar{i} \leq m^{\bar{l}}}\left\|\sum_{\overline{1} \leq \bar{j} \leq \bar{i}} \eta_{\bar{l}, \bar{j}}\right\|^{p}\right)^{1 / p} \leq c\left(\mathbb{E}\left\|\sum_{\overline{1} \leq \bar{j} \leq \bar{l}} \eta_{\bar{l}, \bar{j}}\right\|^{p}\right)^{1 / p} .
$$

Moreover, Lemma 1 gives

$$
\left(\mathbb{E}\left\|\sum_{\overline{1} \leq \bar{j} \leq \bar{l}} \eta_{\bar{l}, \bar{j}}\right\|^{p}\right)^{1 / p} \leq 2 \tau_{p}(X)\left(\sum_{\overline{1} \leq \bar{j} \leq \bar{l}} \mathbb{E}\left\|\eta_{\bar{l}, \bar{j}}\right\|^{p}\right)^{1 / p} .
$$

From (33) and (34) we conclude for $p>1$

$$
\left(\mathbb{E} \max _{\overline{1} \leq \bar{i} \leq m^{\bar{l}}}\left\|\sum_{\overline{1} \leq \bar{j} \leq \bar{i}} \eta_{\bar{l}, \bar{j}}\right\|^{p}\right)^{1 / p} \leq c \tau_{p}(X)\left(\sum_{\overline{1} \leq \bar{j} \leq \bar{l}} \mathbb{E}\left\|\eta_{\bar{l}, \bar{j}}\right\|^{p}\right)^{1 / p} .
$$

The same relation also holds for $p=1$ by the triangle inequality. We obtain from (29-30), (32), and (35)

$$
\left(\mathbb{E}\left\|V_{L}^{X} S_{1}^{X} f-A_{n, \omega}^{1,0, X} f\right\|_{C(Q, X)}^{p}\right)^{1 / p} \leq c \tau_{p}(X) m^{-(1-1 / p) L}\|f\|_{C(Q, X)} .
$$

Now relation (26) for $r=0$ follows from (20-21), (27-28), and (36).

As in the proof of Proposition 1 the case $r \geq 1$ follows from the case $r=0$ and (5), since

$$
S_{1}^{X} f-A_{n, \omega}^{1, r, X} f=S_{1}^{X}\left(f-P_{k}^{r, d, X} f\right)-A_{n, \omega}^{1,0, X}\left(f-P_{k}^{r, d, X} f\right) .
$$

By (16-17) and (19-23) the number of function values used in $A_{n, \omega}^{1, r, X} f$ is

$$
c k^{d}+c \sum_{|\bar{l}|=L} m^{l_{1}} \ldots m^{l_{d}} \leq c n
$$


Theorem 1. Let $r \in \mathbb{N}_{0}, \iota \in\{0,1\}, 1 \leq p \leq 2$. Then there are constants $c_{1-4}>0$ such that for all Banach spaces $X$ and $n \in \mathbb{N}$ the following hold. The deterministic $n$-th minimal error satisfies

$$
c_{1} n^{-r / d} \leq e_{n}^{\operatorname{det}}\left(S_{\iota}^{X}, B_{C^{r}(Q, X)}\right) \leq c_{2} n^{-r / d} .
$$

Moreover, if $X$ is of type $p$ and $p_{X}$ is the supremum of all $p_{1}$ such that $X$ is of type $p_{1}$, then the randomized $n$-th minimal errors fulfills

$$
c_{3} n^{-r / d-1+1 / p_{X}} \leq e_{n}^{\mathrm{ran}}\left(S_{\iota}^{X}, B_{C^{r}(Q, X)}\right) \leq c_{4} \tau_{p}(X) n^{-r / d-1+1 / p} .
$$

Proof. The upper bounds follow from Propositions 1 and 2. Since definite integration is a particular case of indefinite integration in the sense that $S_{0}^{X} f=$ $\left(S_{1}^{X} f\right)(\overline{1})$, it suffices to prove the lower bound for $S_{0}^{X}$. The lower bounds for the deterministic setting and for the randomized setting with $p_{X}=2$ follow from the respective scalar cases (6) and (7), since trivially every Banach space $X$ over $\mathbb{K}$ contains an isometric copy of $\mathbb{K}$.

It remains to show the lower bound for the randomized setting for Banach spaces with $p_{X}<2$. Any such Banach space must be infinite dimensional (a finite dimensional space $X$ always has $p_{X}=2$ ). Let $n \in \mathbb{N}$ and let $k \in \mathbb{N}$ be such that

$$
(k-1)^{d}<8 n \leq k^{d} .
$$

The Maurey-Pisier Theorem (see [9], Th. 2.3) implies that for every $k \in \mathbb{N}$ there is a subspace $E_{k} \subset X$ of dimension $k^{d}$ and an isomorphism $T: \ell_{p_{X}}^{k^{d}} \rightarrow E_{k}$ with $\|T\| \leq 1$ and $\left\|T^{-1}\right\| \leq 2$. Let $x_{i}=T e_{i}$, where $\left(e_{i}\right)_{i=1}^{k^{d}}$ is the unit vector basis of $\ell_{p_{X}}^{k^{d}}$. Let $\psi \in C^{\infty}\left(\mathbb{R}^{d}\right)$ be such that $\psi(t)>0$ for $t \in(0,1)^{d}$ and $\operatorname{supp} \psi \subset[0,1]^{d}$. Let $\left(Q_{i}\right)_{i=1}^{k^{d}}$ be the partition of $Q$ into closed cubes of side length $k^{-1}$ of disjoint interior, let $t_{i}$ be the point in $Q_{i}$ with minimal coordinates and define $\psi_{i} \in C(Q)$ by

$$
\psi_{i}(t)=\psi\left(k\left(t-t_{i}\right)\right) \quad\left(i=1, \ldots, k^{d}\right) .
$$

It is readily checked that there is a constant $c_{0}>0$ such that for all $\left(\alpha_{i}\right)_{i=1}^{k^{d}} \in$ $[-1,1]^{k^{d}}$

$$
c_{0} k^{-r} \sum_{i=1}^{k^{d}} \alpha_{i} x_{i} \psi_{i} \in B_{C^{r}(Q, X)} .
$$

Put $f_{i}=c_{0} k^{-r} x_{i} \psi_{i}$ and $\sigma=\int_{Q} \psi(t) d t$. Then for $\left(\alpha_{i}\right)_{i=1}^{k^{d}} \in \mathbb{R}^{k^{d}}$

$$
\begin{gathered}
\left\|\sum_{i=1}^{k^{d}} \alpha_{i} S_{0}^{X} f_{i}\right\|=c_{0} k^{-r}\left\|\sum_{i=1}^{k^{d}} \alpha_{i} x_{i} \int_{Q} \psi_{i}(t) d t\right\| \\
=c_{0} \sigma k^{-r-d}\left\|\sum_{i=1}^{k^{d}} \alpha_{i} x_{i}\right\| \geq c k^{-r-d}\left(\sum_{i=1}^{k^{d}}\left|\alpha_{i}\right|^{p_{X}}\right)^{1 / p_{X}} .
\end{gathered}
$$


Next we use Lemma 5 and 6 of [3] with $K=X$ (Lemma 6 is formulated for $K=\mathbb{R}$, but directly carries over to $K=X$ ) and (37) to obtain

$$
\begin{aligned}
e_{n}^{\mathrm{ran}}\left(S_{0}^{X}, B_{C^{r}(Q, X)}\right) & \geq \frac{1}{4} \min _{I \subseteq\left\{1, \ldots, k^{d}\right\},|I| \geq k^{d}-4 n} \mathbb{E}\left\|\sum_{i \in I} \varepsilon_{i} S_{0}^{X} f_{i}\right\| \\
& \geq c k^{-r-\left(1-1 / p_{X}\right) d} \geq c n^{-r / d-1+1 / p_{X}},
\end{aligned}
$$

where $\left(\varepsilon_{i}\right)_{i=1}^{k^{d}}$ is a sequence of independent centered Bernoulli random variables.

Note that the bounds in the randomized cases of Theorem 1 are matching up to an arbitrarily small gap in the exponent. In some cases, they are even of matching order.

Corollary 1. Let $r \in \mathbb{N}_{0}, 1 \leq p \leq 2, \iota \in\{0,1\}$. Then there are constants $c_{1}, c_{2}>0$ such that the following hold. Let $X$ be a Banach space which is of type $p$ and moreover, satisfies $p_{X}=p$ (that is, the supremum of types is attained). Then for all $n \in \mathbb{N}$

$$
c_{1} n^{-r / d-1+1 / p} \leq e_{n}^{\mathrm{ran}}\left(S_{\iota}^{X}, B_{C^{r}(Q, X)}\right) \leq c_{2} \tau_{p}(X) n^{-r / d-1+1 / p} .
$$

This holds, in particular, for spaces of type 2 with $p=2$ and, if $1 \leq p_{1}<\infty$, for spaces $X=L_{p_{1}}(\mathcal{N}, \nu)$, where $(\mathcal{N}, \nu)$ is some measure space, with $p=\min \left(p_{1}, 2\right)$.

For general Banach spaces $X$ upper and lower bounds of matching order of $e_{n}^{\mathrm{ran}}\left(S_{\iota}^{X}, B_{C^{r}(Q, X)}\right)(\iota=0,1)$ remain an open problem.

\section{A multilevel procedure}

In the previous section we considered Banach space valued information functionals. Now we develop a scheme which will serve as a bridge between the Banach space and the scalar case. It is based on the multilevel Monte Carlo approach from $[2,6]$. Assume that a Banach space $Y$ is continuously embedded into the Banach space $X$, and let $J$ be the embedding map. We shall identify elements of $Y$ with their images in $X$. For $r, \varrho \in \mathbb{N}_{0}$ we consider integration of functions from the set

$$
B_{C^{r}(Q, X)} \cap B_{C \varrho(Q, Y)} .
$$

Let $\left(T_{l}\right)_{l=0}^{\infty} \subset \mathscr{L}(X)$ (this is intended to be a sequence which approximates the embedding $J$ ) and set for $l \in \mathbb{N}_{0}$

$$
R_{l}=T_{l} \otimes I_{C(Q)} \in \mathscr{L}(C(Q, X)) .
$$

The operator $R_{l}$ is just the pointwise application of $T_{l}$ in the sense that for $f \in C(Q, X)$ and $t \in Q$ we have $\left(R_{l} f\right)(t)=T_{l} f(t)$. Fix any $l_{0}, l_{1} \in \mathbb{N}_{0}, l_{0} \leq l_{1}$, 
$n_{l_{0}}, \ldots, n_{l_{1}} \in \mathbb{N}$ and define for $\iota \in\{0,1\}$ and $f \in C(Q, X)$ an approximation $A_{\omega}^{(\iota)} f$ to $S_{\iota}^{X} f$ as follows:

$$
A_{\omega}^{(\iota)} f=A_{n_{l_{0}}, \omega}^{\iota, r, X} R_{l_{0}} f+\sum_{l=l_{0}+1}^{l_{1}} A_{n_{l}, \omega}^{\iota, \varrho, X}\left(R_{l}-R_{l-1}\right) f
$$

and $A^{(\iota)}=\left(A_{\omega}^{(\iota)}\right)_{\omega \in \Omega}$. It follows from (13), (24), and (38) that

$$
A_{\omega}^{(\iota)}=T_{l_{0}} \otimes A_{n_{l_{0}}, \omega}^{\iota, r}+\sum_{l=l_{0}+1}^{l_{1}}\left(T_{l}-T_{l-1}\right) \otimes A_{n_{l}, \omega}^{\iota, \varrho} .
$$

Furthermore, put

$$
X_{l}=\operatorname{cl}_{X}\left(T_{l}(X)\right) \quad\left(l \in \mathbb{N}_{0}\right), \quad X_{l-1, l}=\operatorname{cl}_{X}\left(\left(T_{l}-T_{l-1}\right)(X)\right) \quad(l \in \mathbb{N}),
$$

where $\mathrm{cl}_{X}$ denotes the closure in $X$. In particular, $X_{l}$ and $X_{l-1, l}$ are endowed with the norm induced by $X$. Given a Banach space $Z$, we introduce the notation $G_{0}(Z)=Z$ and $G_{1}(Z)=C(Q, Z)$. Now we estimate the error of $A_{\omega}^{(\iota)}$ on $B_{C^{r}(Q, X)} \cap$ $B_{C e(Q, Y)}$.

Proposition 3. Let $1 \leq p \leq 2, r, \varrho \in \mathbb{N}_{0}$, and $\iota \in\{0,1\}$. Then there are constants $c_{1}, c_{2}>0$ such that for all Banach spaces $X, Y$, and operators $\left(T_{l}\right)_{l=0}^{\infty}$ as above, for all $l_{0}, l_{1} \in \mathbb{N}_{0}$ with $l_{0} \leq l_{1}$, and for all $\left(n_{l}\right)_{l=l_{0}}^{l_{1}} \subset \mathbb{N}$ the so-defined algorithm $A_{\omega}^{(\iota)}$ satisfies

$$
\begin{aligned}
& \sup _{f \in B_{C^{r}(Q, X) \cap B_{C^{\varrho}(Q, Y)}}\left\|S_{\iota}^{X} f-A_{\omega}^{(\iota)} f\right\|_{G_{\iota}(X)}} \leq \quad\left\|J-T_{l_{1}} J\right\|_{\mathscr{L}(Y, X)}+c_{1}\left\|T_{l_{0}}\right\|_{\mathscr{L}(X)} n_{l_{0}}^{-r / d} \\
& \leq \quad+c_{1} \sum_{l=l_{0}+1}^{l_{1}}\left\|\left(T_{l}-T_{l-1}\right) J\right\|_{\mathscr{L}(Y, X)} n_{l}^{-\varrho / d} \quad(\omega \in \Omega)
\end{aligned}
$$

and

$$
\begin{aligned}
& \sup _{f \in B_{C^{r}(Q, X)} \cap B_{C} \varrho(Q, Y)}\left(\mathbb{E}\left\|S_{\iota}^{X} f-A_{\omega}^{(\iota)} f\right\|_{G_{\iota}(X)}^{p}\right)^{1 / p} \\
& \leq\left\|J-T_{l_{1}} J\right\|_{\mathscr{L}(Y, X)}+c_{2} \tau_{p}\left(X_{l_{0}}\right)\left\|T_{l_{0}}\right\|_{\mathscr{L}(X)} n_{l_{0}}^{-r / d-1+1 / p} \\
& \quad+c_{2} \sum_{l=l_{0}+1}^{l_{1}} \tau_{p}\left(X_{l-1, l}\right)\left\|\left(T_{l}-T_{l-1}\right) J\right\|_{\mathscr{L}(Y, X)} n_{l}^{-\varrho / d-1+1 / p} .
\end{aligned}
$$

Proof. Let $f \in B_{C^{r}(Q, X)} \cap B_{C \varrho(Q, Y)}$. From (39) we get

$$
\begin{aligned}
& \left\|S_{\iota}^{X} f-A_{\omega}^{(\iota)} f\right\|_{G_{\iota}(X)} \\
& \leq\left\|S_{\iota}^{X} f-S_{\iota}^{X} R_{l_{1}} f\right\|_{G_{\iota}(X)}+\left\|S_{\iota}^{X} R_{l_{0}} f-A_{n_{l_{0}}, \omega}^{\iota, r, X} R_{l_{0}} f\right\|_{G_{\iota}\left(X_{l_{0}}\right)} \\
& \quad+\sum_{l=l_{0}+1}^{l_{1}}\left\|S_{\iota}^{X}\left(R_{l}-R_{l-1}\right) f-A_{n_{l}, \omega}^{\iota, \varrho, X}\left(R_{l}-R_{l-1}\right) f\right\|_{G_{\iota}\left(X_{l-1, l}\right)} .
\end{aligned}
$$


We have

$$
\begin{aligned}
& \left\|S_{\iota}^{X} f-S_{\iota}^{X} R_{l_{1}} f\right\|_{G_{\iota}(X)} \leq\left\|S_{\iota}^{X}\right\|_{\mathscr{L}\left(C(Q, X), G_{\iota}(X)\right)}\left\|f-R_{l_{1}} f\right\|_{C(Q, X)} \\
\leq & \left\|J-T_{l_{1}} J\right\|_{\mathscr{L}(Y, X)}\|f\|_{C(Q, Y)} \leq\left\|J-T_{l_{1}} J\right\|_{\mathscr{L}(Y, X) .}
\end{aligned}
$$

Furthermore, by Propositions 1 and 2

$$
\begin{aligned}
& \left\|S_{\iota}^{X} R_{l_{0}} f-A_{n_{l_{0}}, \omega}^{\iota, r, X} R_{l_{0}} f\right\|_{G_{\iota}\left(X_{l_{0}}\right)} \leq c n_{l_{0}}^{-r / d}\left\|R_{l_{0}} f\right\|_{C^{r}\left(Q, X_{l_{0}}\right)} \\
\leq & c n_{l_{0}}^{-r / d}\left\|T_{l_{0}}\right\|_{\mathscr{L}(X)}\|f\|_{C^{r}(Q, X)} \leq c\left\|T_{l_{0}}\right\|_{\mathscr{L}(X)} n_{l_{0}}^{-r / d}
\end{aligned}
$$

and similarly,

$$
\mathbb{E}\left(\left\|S_{\iota}^{X} R_{l_{0}} f-A_{n_{l_{0}}, \omega}^{\iota, r, X} R_{l_{0}} f\right\|_{G_{\iota}\left(X_{l_{0}}\right)}^{p}\right)^{1 / p} \leq c \tau_{p}\left(X_{l_{0}}\right)\left\|T_{l_{0}}\right\|_{\mathscr{L}(X)} n_{l_{0}}^{-r / d-1+1 / p}
$$

For $l_{0}<l \leq l_{1}$ we obtain

$$
\begin{aligned}
& \left\|S_{\iota}^{X}\left(R_{l}-R_{l-1}\right) f-A_{n_{l}, \omega}^{\iota, \varrho, X}\left(R_{l}-R_{l-1}\right) f\right\|_{G_{\iota}\left(X_{l-1, l}\right)} \\
& \quad \leq c n_{l}^{-\varrho / d}\left\|\left(R_{l}-R_{l-1}\right) f\right\|_{C \varrho\left(Q, X_{l-1, l}\right)} \\
& \quad \leq c n_{l}^{-\varrho / d}\left\|\left(T_{l}-T_{l-1}\right) J\right\|_{\mathscr{L}(Y, X)}\|f\|_{C}^{\varrho(Q, Y)} \leq c\left\|\left(T_{l}-T_{l-1}\right) J\right\|_{\mathscr{L}(Y, X)} n_{l}^{-\varrho / d}(48)
\end{aligned}
$$

and

$$
\begin{aligned}
& \mathbb{E}\left(\left\|S_{\iota}^{X}\left(R_{l}-R_{l-1}\right) f-A_{n_{l}, \omega}^{\iota, \varrho, X}\left(R_{l}-R_{l-1}\right) f\right\|_{G_{\iota}\left(X_{l-1, l}\right)}^{p}\right)^{1 / p} \\
& \leq c \tau_{p}\left(X_{l-1, l}\right)\left\|\left(T_{l}-T_{l-1}\right) J\right\|_{\mathscr{L}(Y, X)} n_{l}^{-\varrho / d-1+1 / p} .
\end{aligned}
$$

Combining (44-49) yields the result.

\section{Scalar parametric case}

In this section we apply the previous results to parametric definite and indefinite integration. Let $d, d_{1} \in \mathbb{N}, Q_{1}=[0,1]^{d_{1}}$. We consider numerical integration of functions depending on a parameter $s \in Q_{1}$. The definite parametric integration operator $\mathscr{S}_{0}: C\left(Q_{1} \times Q\right) \rightarrow C\left(Q_{1}\right)$ is given by

$$
\left(\mathscr{S}_{0} f\right)(s)=\int_{Q} f(s, t) d t \quad\left(s \in Q_{1}\right) .
$$

We put $F=B_{C^{r}\left(Q_{1} \times Q\right)}$, the set $K$ is the scalar field $\mathbb{K}$, and $\Lambda$ is the following class of information functionals $\Lambda\left(Q_{1} \times Q, \mathbb{K}\right)=\left\{\delta_{s, t}: s \in Q_{1}, t \in Q\right\}$ where $\delta_{s, t}(f)=f(s, t)$. This is just standard information consisting of values of $f$. Hence, the definite parametric integration problem is

$$
\Pi_{0}=\left(B_{C^{r}\left(Q_{1} \times Q\right)}, C\left(Q_{1}\right), \mathscr{S}_{0}, \mathbb{K}, \Lambda\left(Q_{1} \times Q, \mathbb{K}\right)\right) .
$$


The indefinite parametric integration operator $\mathscr{S}_{1}: C\left(Q_{1} \times Q\right) \rightarrow C\left(Q_{1} \times Q\right)$ is given by

$$
\left(\mathscr{S}_{1} f\right)(s, t)=\int_{[0, t]} f(s, u) d u \quad\left(s \in Q_{1}, t \in Q\right) .
$$

Here $F, K, \Lambda$ are chosen to be the same as above, so the indefinite parametric integration problem is described by

$$
\Pi_{1}=\left(B_{C^{r}\left(Q_{1} \times Q\right)}, C\left(Q_{1} \times Q\right), \mathscr{S}_{1}, \mathbb{K}, \Lambda\left(Q_{1} \times Q, \mathbb{K}\right)\right) .
$$

We can relate these problems to the previously considered Banach space valued ones as follows. Setting $X=C\left(Q_{1}\right)$, we have

$$
C\left(Q_{1} \times Q\right)=C\left(Q_{1}\right) \otimes_{\lambda} C(Q)=X \otimes_{\lambda} C(Q)=C(Q, X)
$$

and $\mathscr{S}_{\iota}=S_{\iota}^{C\left(Q_{1}\right)}(\iota=0,1)$. Moreover, referring to the notation of Section 4 , we put $Y=C^{r}\left(Q_{1}\right)$ and $\varrho=0$, which gives

$$
B_{C^{r}\left(Q_{1} \times Q\right)} \subseteq B_{C^{r}\left(Q, C\left(Q_{1}\right)\right)} \cap B_{C\left(Q, C^{r}\left(Q_{1}\right)\right)}=B_{C^{r}(Q, X)} \cap B_{C(Q, Y)} .
$$

Let $r_{1}=\max (r, 1)$ and define for $l \in \mathbb{N}_{0}$

$$
T_{l}=P_{2^{l}}^{r_{1}, d_{1}} \in \mathscr{L}\left(C\left(Q_{1}\right)\right) .
$$

By (5),

$$
\left\|T_{l}\right\|_{\mathscr{L}\left(C\left(Q_{1}\right)\right)} \leq c_{1}, \quad\left\|J-T_{l} J\right\|_{\mathscr{L}\left(C^{r}\left(Q_{1}\right), C\left(Q_{1}\right)\right)} \leq c_{2} 2^{-r l},
$$

where $J: C^{r}\left(Q_{1}\right) \rightarrow C\left(Q_{1}\right)$ is the embedding. The algorithms $A_{\omega}^{(\iota)}$ defined in (39) and equivalently (40) turn into

$$
A_{\omega}^{(\iota)}=P_{2^{l_{0}}}^{r_{1}, d_{1}} \otimes A_{n_{l_{0}, \omega}}^{\iota, r}+\sum_{l=l_{0}+1}^{l_{1}}\left(P_{2^{l}}^{r_{1}, d_{1}}-P_{2^{l-1}}^{r_{1}, d_{1}}\right) \otimes A_{n_{l}, \omega}^{\iota, 0} .
$$

Let us note that (52) together with the definitions of $P_{m}^{r_{1}, d_{1}}$ and $A_{n, \omega}^{\iota, r}$ imply the following representation of $A_{\omega}^{(\iota)}$. There are $s_{l, i} \in Q_{1}, t_{l, j, \omega} \in Q, \varphi_{l, i} \in C\left(Q_{1}\right)$, $\psi_{l, j, \omega}^{(\iota)} \in \mathbb{K}$ if $\iota=0, \psi_{l, j, \omega}^{(\iota)} \in C(Q)$ if $\iota=1, M_{l} \leq c 2^{d_{1} l}$, and $N_{l} \leq c n_{l}$ such that

$$
A_{\omega}^{(\iota)} f=\sum_{l=l_{0}}^{l_{1}} \sum_{i=1}^{M_{l}} \sum_{j=1}^{N_{l}} f\left(s_{l, i}, t_{l, j, \omega}\right) \varphi_{l, i} \otimes \psi_{l, j, \omega}^{(\iota)} \quad\left(f \in C\left(Q_{1} \times Q\right), \omega \in \Omega\right) .
$$

The particular shape of these functions can be read from the definitions (11-12) and (16-23), for more details in the case $\iota=1$ see also [5]. It follows that

$$
\operatorname{card}\left(A_{\omega}^{(\iota)}\right) \leq c \sum_{l=l_{0}}^{l_{1}} n_{l} 2^{d_{1} l} \quad(\omega \in \Omega) .
$$

Now we estimate the error of $A_{\omega}^{(\iota)}$. Recall the notation $G_{0}\left(C\left(Q_{1}\right)\right)=C\left(Q_{1}\right)$ and $G_{1}\left(C\left(Q_{1}\right)\right)=C\left(Q_{1} \times Q\right)$. 
Proposition 4. Let $r \in \mathbb{N}_{0}, d, d_{1} \in \mathbb{N}, \iota \in\{0,1\}$. There are constants $c_{1-4}>0$ such that the following hold. For each $n \in \mathbb{N}$ there are $l_{0} \in \mathbb{N}_{0}$ and $n_{l_{0}} \in \mathbb{N}$ such that with $l_{1}=l_{0}$ we have $\operatorname{card}\left(A_{\omega}^{(\iota)}\right) \leq c_{1} n$ and

$$
\sup _{f \in B_{C^{r}\left(Q_{1} \times Q\right)}}\left\|\mathscr{S}_{\iota} f-A_{\omega}^{(\iota)} f\right\|_{G_{\iota}\left(C\left(Q_{1}\right)\right)} \leq c_{2} n^{-\frac{r}{d_{1}+d}}
$$

for all $\omega \in \Omega$. Moreover, for each $n \in \mathbb{N}$ with $n \geq 2$ there is a choice of $l_{0}, l_{1} \in \mathbb{N}_{0}$, $\left(n_{l}\right)_{l=l_{0}}^{l_{1}} \subset \mathbb{N}$ such that $\operatorname{card}\left(A_{\omega}^{(\iota)}\right) \leq c_{3} n(\omega \in \Omega)$ and

$$
\begin{aligned}
\sup _{f \in B_{C^{r}\left(Q_{1} \times Q\right)}}\left(\mathbb{E}\left\|\mathscr{S}_{\iota} f-A_{\omega}^{(\iota)} f\right\|_{G_{\iota}\left(C\left(Q_{1}\right)\right)}^{2}\right)^{1 / 2} \\
\leq c_{4}\left\{\begin{array}{lll}
n^{-\frac{2 r+d}{2\left(d_{1}+d\right)}}(\log n)^{\frac{1}{2}} & \text { if } r / d_{1}>1 / 2 \\
n^{-\frac{1}{2}(\log n)^{2}} & \text { if } & r / d_{1}=1 / 2 \\
n^{-\frac{r}{d_{1}}}(\log n)^{\frac{r}{d_{1}}} & \text { if } & r / d_{1}<1 / 2 .
\end{array}\right.
\end{aligned}
$$

Proof. Let $n \in \mathbb{N}$ and put

$$
l^{*}=\left\lceil\frac{\log _{2} n}{d_{1}}\right\rceil, \quad l_{0}=\left\lfloor\frac{d_{1}}{d_{1}+d} l^{*}\right\rfloor .
$$

Furthermore, let $l_{1} \in \mathbb{N}_{0}, l_{0} \leq l_{1} \leq l^{*}, \delta_{0}, \delta_{1} \geq 0$ to be fixed later on and define

$$
n_{l_{0}}=2^{d_{1}\left(l^{*}-l_{0}\right)}, \quad n_{l}=\left\lceil 2^{d_{1}\left(l^{*}-l\right)-\delta_{0}\left(l-l_{0}\right)-\delta_{1}\left(l_{1}-l\right)}\right\rceil \quad\left(l=l_{0}+1, \ldots, l_{1}\right) .
$$

Then by (54) the cost fulfills

$$
\begin{aligned}
\operatorname{card}\left(A_{\omega}^{(\iota)}\right) & \leq c \sum_{l=l_{0}}^{l_{1}} n_{l} 2^{d_{1} l} \leq c 2^{d_{1} l^{*}}+c \sum_{l=l_{0}+1}^{l_{1}} 2^{d_{1} l^{*}-\delta_{0}\left(l-l_{0}\right)-\delta_{1}\left(l_{1}-l\right)} \\
& \leq c \begin{cases}n & \text { if } \delta_{0}>0 \text { or } \delta_{1}>0 \\
n & \text { if } \delta_{0}=\delta_{1}=0 \text { and } l_{1}=l_{0} \\
n \log n & \text { if } \delta_{0}=\delta_{1}=0 \text { and } l_{1}>l_{0} .\end{cases}
\end{aligned}
$$

To show (55), we put $l_{1}=l_{0}$ and get from (42) of Proposition 3, (51), and $(57-58)$

$$
\begin{aligned}
& \sup _{f \in B_{C^{r}\left(Q_{1} \times Q\right)}}\left\|\mathscr{S}_{\iota} f-A_{\omega}^{(\iota)} f\right\|_{G_{\iota}\left(C\left(Q_{1}\right)\right)} \\
& \leq c 2^{-r l_{0}}+c n_{l_{0}}^{-\frac{r}{d}} \leq c 2^{-r \frac{d_{1}}{d+d_{1}} l^{*}}+c 2^{-\frac{r}{d} d_{1}\left(l^{*}-l_{0}\right)} \leq c 2^{-\frac{r d_{1} l^{*}}{d+d_{1}}} \leq c n^{-\frac{r}{d+d_{1}}}
\end{aligned}
$$

which together with (59) gives (55).

Now we turn to the proof of (56) and assume, in addition, that $n \geq 2$. Observe that by (41) and (50)

$$
X_{l}=P_{2^{l}}^{r_{1}, d_{1}}\left(C\left(Q_{1}\right)\right)=P_{2^{l}}^{r_{1}, d_{1}}\left(\ell_{\infty}\left(\Gamma_{r_{1} 2^{l}}^{d_{1}}\right)\right)
$$


and $P_{2^{l}}^{r_{1}, d_{1}}: \ell_{\infty}\left(\Gamma_{r_{1} 2^{l}}^{d_{1}}\right) \rightarrow X_{l}$ is an isomorphism which satisfies

$$
\left\|P_{2^{l}}^{r_{1}, d_{1}}\right\| \leq c_{1}, \quad\left\|\left(P_{2^{l}}^{r_{1}, d_{1}}\right)^{-1}\right\|=1
$$

Indeed, the first estimate is just the first part of (5), the second estimate is a consequence of the fact that the inverse of the interpolation operator is just the restriction of functions in $X_{l}$ to $\Gamma_{r_{1} 2^{l}}^{d_{1}}$. It follows that

$$
\tau_{2}\left(X_{l}\right) \leq c \tau_{2}\left(\ell_{\infty}\left(\Gamma_{r_{1} 2^{l}}^{d_{1}}\right)\right) \leq c(l+1)^{1 / 2} .
$$

By (60), $X_{l-1} \subseteq X_{l}$ for $l \geq 1$, therefore (41) implies that we also have $X_{l-1, l} \subseteq X_{l}$, thus

$$
\tau_{2}\left(X_{l-1, l}\right) \leq c(l+1)^{1 / 2}
$$

For brevity we denote

$$
E:=\sup _{f \in B_{C^{r}\left(Q_{1} \times Q\right)}}\left(\mathbb{E}\left\|\mathscr{S}_{\iota} f-A_{\omega}^{(\iota)} f\right\|_{G_{\iota}\left(C\left(Q_{1}\right)\right)}^{2}\right)^{1 / 2} .
$$

By (43) of Proposition 3, (51), and (61-62)

$$
\begin{aligned}
E & \leq c 2^{-r l_{1}}+c\left(l_{0}+1\right)^{1 / 2} n_{l_{0}}^{-r / d-1 / 2}+c \sum_{l=l_{0}+1}^{l_{1}}(l+1)^{1 / 2} 2^{-r l} n_{l}^{-1 / 2} \\
& \leq c 2^{-r l_{1}}+c\left(l^{*}+1\right)^{1 / 2} 2^{-(r / d+1 / 2) d_{1}\left(l^{*}-l_{0}\right)}+c\left(l^{*}+1\right)^{1 / 2} \sum_{l=l_{0}+1}^{l_{1}} 2^{-\mu(l)}
\end{aligned}
$$

where we defined

$$
\mu(l)=r l+\left(d_{1}\left(l^{*}-l\right)-\delta_{0}\left(l-l_{0}\right)-\delta_{1}\left(l_{1}-l\right)\right) / 2 \quad\left(l_{0} \leq l \leq l_{1}\right) .
$$

We have from (57)

$$
\frac{r d_{1}}{d}\left(l^{*}-l_{0}\right) \geq \frac{r d_{1}}{d} \cdot \frac{d}{d_{1}+d} l^{*}=r \frac{d_{1}}{d_{1}+d} l^{*} \geq r l_{0},
$$

consequently,

$$
2^{-(r / d+1 / 2) d_{1}\left(l^{*}-l_{0}\right)} \leq c 2^{-r l_{0}-d_{1}\left(l^{*}-l_{0}\right) / 2} \leq c 2^{-\mu\left(l_{0}\right)},
$$

which together with (63) gives

$$
E \leq c 2^{-r l_{1}}+c\left(l^{*}+1\right)^{1 / 2} \sum_{l=l_{0}}^{l_{1}} 2^{-\mu(l)} .
$$

We rewrite (64) as

$$
\mu(l)=r l_{0}+d_{1}\left(l^{*}-l_{1}\right) / 2+\left(r-\delta_{0} / 2\right)\left(l-l_{0}\right)+\left(d_{1}-\delta_{1}\right)\left(l_{1}-l\right) / 2 .
$$


If $r>d_{1} / 2$, we set $\delta_{1}=0, l_{1}=l^{*}$ and choose $\delta_{0}>0$ in such a way that $r-\delta_{0} / 2>d_{1} / 2$. From (57), (65), and (66) we obtain

$$
\begin{aligned}
E & \leq c 2^{-r l^{*}}+c\left(l^{*}+1\right)^{1 / 2} \sum_{l=l_{0}}^{l^{*}} 2^{-r l_{0}-\left(r-\delta_{0} / 2\right)\left(l-l_{0}\right)-d_{1}\left(l^{*}-l\right) / 2} \\
& \leq c 2^{-r l^{*}}+c\left(l^{*}+1\right)^{1 / 2} 2^{-r l_{0}-d_{1}\left(l^{*}-l_{0}\right) / 2} \\
& \leq c 2^{-r l^{*}}+c\left(l^{*}+1\right)^{1 / 2} 2^{-\frac{(r+d / 2) d_{1}}{d_{1}+d} l^{*}} \\
& \leq c\left(l^{*}+1\right)^{1 / 2} 2^{-\frac{(r+d / 2) d_{1}}{d_{1}+d} l^{*}} \leq c n^{-\frac{r+d / 2}{d_{1}+d}}(\log n)^{1 / 2}
\end{aligned}
$$

where in the step from (67) to $(68)$ we used $\frac{(r+d / 2) d_{1}}{d_{1}+d}<r$, which follows from the assumption $r>d_{1} / 2$. This together with (59) proves (56) for $r>d_{1} / 2$.

If $r=d_{1} / 2$, we set $\delta_{0}=\delta_{1}=0, l_{1}=l^{*}$ and get from (57), (65), and (66)

$$
\begin{aligned}
E & \leq c 2^{-r l^{*}}+c\left(l^{*}+1\right)^{1 / 2} \sum_{l=l_{0}}^{l^{*}} 2^{-r l_{0}-r\left(l-l_{0}\right)-d_{1}\left(l^{*}-l\right) / 2} \\
& \leq c\left(l^{*}+1\right)^{3 / 2} 2^{-d_{1} l^{*} / 2} \leq c n^{-1 / 2}(\log n)^{3 / 2} .
\end{aligned}
$$

Combining this with (59) and transforming $n \log n$ into $n$ gives the respective estimate (56) in this case.

Finally, if $r<d_{1} / 2$, we set $\delta_{0}=0$, choose $\delta_{1}>0$ in such a way that $\left(d_{1}-\right.$ $\left.\delta_{1}\right) / 2>r$ and put

$$
l_{1}=l^{*}-\left\lceil d_{1}^{-1} \log _{2}\left(l^{*}+1\right)\right\rceil
$$

Consequently,

$$
\log _{2}\left(l^{*}+1\right) \leq d_{1}\left(l^{*}-l_{1}\right)<\log _{2}\left(l^{*}+1\right)+d_{1} .
$$

Also observe that there is a constant $c_{0} \in \mathbb{N}$ such that for $n \geq c_{0}$

$$
l_{0} \leq l^{*}-\left\lceil d_{1}^{-1} \log _{2}\left(l^{*}+1\right)\right\rceil \leq l^{*} .
$$

Since for $n<c_{0}$ the estimate (56) follows trivially from (65) by a suitable choice of the constant, we can assume $n \geq c_{0}$, and thus (71). By (57), (65-66), (69), and $(70)$

$$
\begin{aligned}
E & \leq c 2^{-r l_{1}}+c\left(l^{*}+1\right)^{1 / 2} \sum_{l=l_{0}}^{l_{1}} 2^{-r l_{0}-d_{1}\left(l^{*}-l_{1}\right) / 2-r\left(l-l_{0}\right)-\left(d_{1}-\delta_{1}\right)\left(l_{1}-l\right) / 2} \\
& \leq c 2^{-r l_{1}}+c\left(l^{*}+1\right)^{1 / 2} 2^{-r l_{0}-d_{1}\left(l^{*}-l_{1}\right) / 2-r\left(l_{1}-l_{0}\right)} \\
& \leq c 2^{-r l_{1}}+c\left(l^{*}+1\right)^{1 / 2} 2^{-r l_{1}-\left(\log _{2}\left(l^{*}+1\right)\right) / 2} \\
& \leq c 2^{-r l_{1}}=c 2^{-r l^{*}+r\left(l^{*}-l_{1}\right)} \leq c 2^{-r l^{*}+\left(r / d_{1}\right) \log _{2}\left(l^{*}+1\right)} \\
& =c 2^{-r l^{*}}\left(l^{*}+1\right)^{r / d_{1}} \leq c n^{-r / d_{1}}(\log n)^{r / d_{1}} .
\end{aligned}
$$

With this, (56) is now a consequence of (59). 
The following theorem gives the complexity of parametric integration. The case of definite parametric integration is already contained in [6] (with a slightly better upper bound in the limit case $r / d_{1}=1 / 2$ : $(\log n)^{3 / 2}$ instead of $\left.(\log n)^{2}\right)$. The case of indefinite parametric integration is new.

Theorem 2. Let $r \in \mathbb{N}_{0}, d, d_{1} \in \mathbb{N}, \iota \in\{0,1\}$. Then there are constants $c_{1-8}>0$ such that for all $n \in \mathbb{N}$ with $n \geq 2$ the deterministic $n$-th minimal error satisfies

$$
c_{1} n^{-\frac{r}{d_{1}+d}} \leq e_{n}^{\operatorname{det}}\left(\mathscr{S}_{\iota}, B_{C^{r}\left(Q_{1} \times Q\right)}\right) \leq c_{2} n^{-\frac{r}{d_{1}+d}} .
$$

For the randomized $n$-th minimal error we have the following: If $r / d_{1}>1 / 2$, then

$$
c_{3} n^{-\frac{2 r+d}{2\left(d_{1}+d\right)}}(\log n)^{\frac{1}{2}} \leq e_{n}^{\operatorname{ran}}\left(\mathscr{S}_{\iota}, B_{C^{r}\left(Q_{1} \times Q\right)}\right) \leq c_{4} n^{-\frac{2 r+d}{2\left(d_{1}+d\right)}}(\log n)^{\frac{1}{2}}
$$

if $r / d_{1}=1 / 2$, then

$$
c_{5} n^{-\frac{1}{2}}(\log n)^{\frac{1}{2}} \leq e_{n}^{\mathrm{ran}}\left(\mathscr{S}_{\iota}, B_{C^{r}\left(Q_{1} \times Q\right)}\right) \leq c_{6} n^{-\frac{1}{2}}(\log n)^{2}
$$

and if $r / d_{1}<1 / 2$, then

$$
c_{7} n^{-\frac{r}{d_{1}}}(\log n)^{\frac{r}{d_{1}}} \leq e_{n}^{\operatorname{ran}}\left(\mathscr{S}_{\iota}, B_{C^{r}\left(Q_{1} \times Q\right)}\right) \leq c_{8} n^{-\frac{r}{d_{1}}}(\log n)^{\frac{r}{d_{1}}} .
$$

Proof. The upper bounds follow from Proposition 4. For the lower bounds it suffices to consider parametric definite integration. But these are contained in Theorem 2.4 of [6] (note a misprint there, case $r<d_{1} / 2: d_{2}$ is to be replaced by $\left.d_{1}\right)$.

Let us finally note that the choice of $Y=C^{r}\left(Q_{1}\right)$ and $\varrho=0$ in this section was motivated by our application to the class $C^{r}\left(Q_{1} \times Q\right)$, but is, of course, not the only interesting one. We leave other cases to future consideration.

\section{References}

[1] A. Defant, K. Floret, Tensor Norms and Operator Ideals, North Holland, Amsterdam, 1993.

[2] S. Heinrich, Monte Carlo complexity of global solution of integral equations, J. Complexity 14 (1998), 151-175.

[3] S. Heinrich, Monte Carlo approximation of weakly singular integral operators, J. Complexity 22 (2006), 192-219.

[4] S. Heinrich, The randomized information complexity of elliptic PDE, J. Complexity 22 (2006), 220-249. 
[5] S. Heinrich, B. Milla, The randomized complexity of indefinite integration, J. Complexity 27 (2011), 352-382.

[6] S. Heinrich, E. Sindambiwe, Monte Carlo complexity of parametric integration, J. Complexity 15 (1999), 317-341.

[7] M. Ledoux, M. Talagrand, Probability in Banach Spaces, Springer, 1991.

[8] W. A. Light, W. Cheney, Approximation Theory in Tensor Product Spaces, Lecture Notes in Mathematics 1169, Springer-Verlag, Berlin, 1985.

[9] B. Maurey, G. Pisier, Series de variables aléatoires vectorielles independantes et propriétés geométriques des espaces de Banach, Stud. Math. 58, 45-90 (1976).

[10] E. Novak, Deterministic and Stochastic Error Bounds in Numerical Analysis, Lecture Notes in Mathematics 1349, Springer, 1988.

[11] E. Novak, H. Woźniakowski, Tractability of Multivariate Problems, Volume 2, Standard Information for Functionals, European Math. Soc., Zürich, 2010 .

[12] J. F. Traub, G. W. Wasilkowski, and H. Woźniakowski, Information-Based Complexity, Academic Press, New York, 1988. 